\title{
A case of blaNDM-1-positive Salmonella Kottbus, Denmark, November 2020
}

\author{
Hans Linde Nielsen ${ }^{1,2}$, Philip K. Thomsen ${ }^{1}$, Eva Litrup ${ }^{3}$, Mia Torpdahl ${ }^{3}$, Søren Overballe-Petersen ${ }^{3}$, Frank Hansen ${ }^{3}$, Anne \\ Kathrine Schultz Christensen ${ }^{1}$, Henrik Hasman ${ }^{3}$ \\ 1. Department of Clinical Microbiology, Aalborg University Hospital, Aalborg, Denmark \\ 2. Department of Clinical Medicine, Aalborg University, Aalborg, Denmark \\ 3. Department of Microbiology and Infection Control, Statens Serum Institut, Copenhagen, Denmark
}

Correspondence: Hans Linde Nielsen (halin@rn.dk)

Citation style for this article:

Nielsen Hans Linde, Thomsen Philip K., Litrup Eva, Torpdahl Mia, Overballe-Petersen Søren, Hansen Frank, Christensen Anne Kathrine Schultz, Hasman Henrik. A case of blaNDM-1-positive Salmonella Kottbus, Denmark, November 2020. Euro Surveill. 2021;26(26):pii=2100569. https://doi.org/10.2807/1560-7917. ES.2021.26.26.2100569

We present a case of carbapenemase-producing blaNDM-1-positive Salmonella Kottbus in an 82-year-old Danish man. The blaNDM-1 was also identified in Escherichia coli and Citrobacter freundii in the same patient on the same $43 \mathrm{~kb}$ IncN2 plasmid, suggesting in vivo inter-species plasmid transfer. $A$ NCBI BLAST analysis of the plasmid (pAMAo03584 NDM-1) identified 12 highly similar plasmids, all originating from east and south-east Asia. This case could be the first confirmed case of blaNDM-1-positive Salmonella not related to travel outside Europe.

In Denmark, non-typhoidal Salmonella (NTS) is notifiable by the diagnosing laboratory and $S$. enterica subsp. enterica serovar Kottbus is a rare serovar, accounting for ca $1 \%$ of all NTS-cases registered over the past 20 years (https://statistik.ssi.dk). S. Kottbus has been isolated from poultry, cattle, pigs and reptiles [1] and has been identified in several outbreaks [2-5]. Carbapenems are not first-choice drugs for the treatment of Salmonella. However, the emergence of resistance to carbapenems, often last-line antimicrobial agents, is a major concern. In human Salmonella infections, five carbapenemases are of major clinical importance, namely Klebsiella pneumoniae carbapenemases (KPC; class A), New Delhi metallo- $\beta$-lactamase (NDM; class B), Verona integron-encoded metallo- $\beta$-lactamase (VIM; class B), and imipenemase (IMP; class B), and oxacillinases (OXA e.g. OXA-48; class D) [6].

We present a case of an NDM-1 carbapenemase-producing $S$. Kottbus, isolated in a Danish man who did not have travel history outside of Europe.

\section{Case report}

The patient was an 82-year-old man with a recent diagnosis of inoperable lung cancer with no option for chemotherapy. In November 2020, he was hospitalised because of intermittent fever, abdominal pain, and diarrhoea for several weeks. On examination, he had tachycardia (112 beats/minute) and a body temperature of $37.4^{\circ} \mathrm{C}$. Laboratory findings showed leucocytosis (34.1 $\times 10^{9} / \mathrm{L}$; norm: 3.5-10.0) and elevated C-reactive protein $(265 \mathrm{mg} / \mathrm{L}$; norm: $<8.0)$. A computed tomography scan with contrast revealed bowel wall thickening in the left colon, suggestive of an underlying inflammatory or infectious condition. A stool sample taken on the day of admission was positive for Clostridioides difficile toxin B (Xpert C. difficile BT, Cepheid, Sunnyvale, California, United States (US)) and oral metronidazole treatment was initiated. In another stool sample also from the day of admission, Salmonella was isolated by routine methods and antimicrobial susceptibility testing was performed using the European Committee on Antimicrobial Susceptibility Testing (EUCAST) standardised disk diffusion method for Enterobacteral es (Breakpoint table v10.0) [7]. Surprisingly, the isolate was resistant to meropenem (inhibition zone diameter: $11 \mathrm{~mm}$ ) and the NG-Test CARBA 5 (NG Biotech, Guipry, France) [8] was positive for NDM. The stool sample was also plated on chromID CARBA SMART agar (bioMérieux, Marcy l'Etoile, France), showing growth of Escherichia coli and Citrobacter freundii; both isolates were NDM-positive with the NG-Test CARBA 5. The diarrhoea symptoms subsided after a few days and the patient recovered. On day 8 , he was discharged and treatment with oral metronidazole continued for a total of 10 days. After 2 weeks, he died at his home.

A review of the patient's records clarified that, in September 2020, he had been at a resort on the Ionian Sea, Greece, for a week-long holiday. The patient and his partner stayed at an all-inclusive hotel; both had an onset of diarrhoea 2 days after arrival. The patient had many loose stools so he visited an outpatient clinic where he received intravenous rehydration and antibiotic therapy with oral cefuroxime. After the patient returned to Denmark, the diarrhoea symptoms 


\section{FIGURE}

Map of the blaNDM-1-carrying $43 \mathrm{~kb}$ IncN2 plasmid pAMA003584_NDM-1 from an isolate of Salmonella Kottbus, Denmark, November 2020

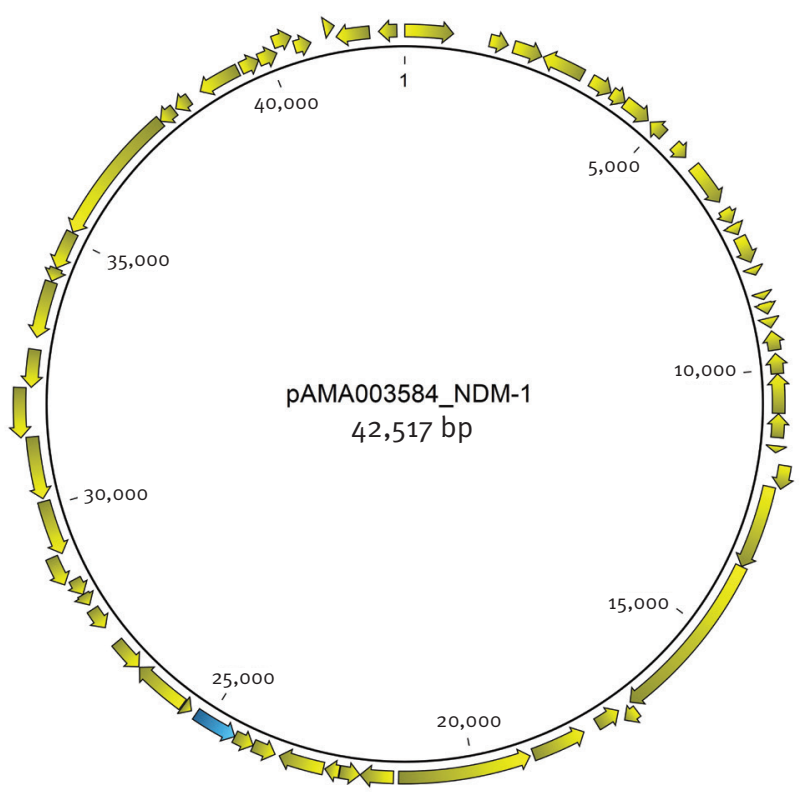

GenBank accession number: MZoo4973. The location of the blaNDM-1 gene is shown in blue.

subsided but the patient was hospitalised a few days later with kidney failure; a rectal swab taken as part of screening procedures for multidrug-resistant bacteria were negative. Throughout September and October, the patient was hospitalised for chronic kidney failure, and was eventually diagnosed with inoperable lung cancer. He was dialysed and received different antibiotic regimens, including oral metronidazole for recurrent diarrhoea. However, a stool sample for enteric pathogenic bacteria was not taken until the final hospital stay in November.

\section{Serotyping and genomic analysis at the National Reference Laboratory}

In 2018, the Danish Health Authority added carbapenemase-producing organisms (CPO) to the list of notifiable bacteria, and the Danish National Reference Laboratory (Statens Serum Institut (SSI)) carries out whole genome sequencing of all CPO isolates. At SSI, the isolate from the patient was serotyped as $S$. Kottbus based on the Kauffmann-White-Le Minor scheme, which was later verified using the sequence data to predict the serotype.

For short-read sequencing of the NDM-1producing $S$. Kottbus, E. coli and $C$. freundii, DNA was extracted using DNeasy Blood and Tissue Kit (Qiagen, Hilden, Germany) and Nextera XT DNA Library Preparation Kit (Illumina, San Diego, California, US) was used before sequencing with a $2 \times 151$ bp pairedend Mid-Output kit (Illumina). The sequence reads are available from the European Nucleotide Archive (ENA; accession number: ERS6246687). For nanopore sequencing, DNA was extracted with the GenFind v3 (Beckman Coulter, Indianapolis, Indiana, US) using a DynaMag-2 magnet (Thermo Fisher Scientific, Waltham, Massachusetts, US). A library was prepared using the Rapid Barcoding Sequencing Kit (SQK-RBKoo4) and sequenced in a R10.3 flow cell (FLO-MIN111) with a MinION Mk1B (Oxford Nanopore Technologies, Oxford, United Kingdom (UK)). Using Guppy v4.2.2 (Oxford Nanopore Technologies), raw fast5 reads were basecalled to fasta format in 'high-accuracy' configuration, demultiplexed and quality filtered to minimum q8. Then Illumina-Nanopore hybrid de novo genome assembly was run with Unicycler vo.4.8-beta [9].

The E. coli was identified as sequence type (ST)399 and $C$. freundii as ST18. The blaNDM-1 was identified in all three isolates placed on the same $43 \mathrm{~kb}$ IncN2 plasmid (pAMAoo3584_NDM-1; GenBank accession number: MZo04973), identified by PlasmidFinder v2.1 [10], as shown in Figure 1.

A National Center for Biotechnology Information (NCBI) Basic Local Alignment Tool (BLAST) analysis of pAMA003584_NDM-1 identified 12 highly similar plasmids, all originating from east and south-east Asia (Table 1).

\section{Ethical Statement}

A signed informed consent for publication from the deceased's partner was obtained before submitting.

\section{Discussion}

In the European Union (EU)/European Economic Area (EEA), the notification rate of NTS was 20 cases per 100,000 inhabitants in 2019 [11]. In Denmark, the notification rate for NTS was almost identical (19.3 cases/100,000 inhabitants) in 2019, while the lowest rates were reported by Cyprus, Greece, Ireland, Italy, Portugal, and Romania ( $\leq 7.1$ cases/100,000 inhabitants) [11].

Here we present the first confirmed case of blaNDM1-positive $S$. Kottbus not related to travel outside Europe. The blaNDM-1 gene was also identified in $E$. coli and $C$. freundii on the same $43 \mathrm{~kb}$ IncN2 plasmid, suggesting an inter-species transfer of the blaNDM1-carrying IncN2 plasmid in vivo.

The first NDM-producing NTS case, published in 2011, was a 60-year-old American man who was transferred from India to a hospital in the US where blaNDM1-positive Salmonella Senftenberg was isolated from a perirectal surveillance culture [12]. Following this, other blaNDM-1-positive human NTS cases were described in connection to India, Pakistan and China, as reviewed by Fernández et al [6]. In 2015, Day et al. reported an isolate of $S$. Senftenberg from the UK, obtained in 2008 from faeces in an outpatient with unknown travel history. The isolate was resistant to 
TABLE 1.

A NCBI BLAST analysis of the blaNDM-1-carrying $43 \mathrm{~kb}$ IncN2 plasmid identified 12 highly similar plasmids all originating from east and south-east Asia

\begin{tabular}{|l|c|c|c|c|}
\hline Plasmid & Organism & Accession length (bp) & GenBank accession number & Country of origin \\
\hline pJN24NDM1 & Escherichia coli strain JN24 & 41,190 & MK368725.1 & China \\
\hline pC2972-5-NDM & Klebsiella pneumoniae strain C2972 & 51,995 & CP039806.1 & China \\
\hline pCRE1.4 & Escherichia coli strain CRE1 & 41,185 & CP034398.1 & Thailand \\
\hline pNH25.5 & Klebsiella pneumoniae strain NH25 & 38,383 & CP024879.1 & Thailand \\
\hline pNDM-ECS01 & Escherichia coli strain ECS01 & 41,190 & KJ413946.1 \\
\hline pC057_NDM1 DNA & Klebsiella pneumoniae C057 & 41,181 & LC521837.1 & Thailand \\
\hline pC099_NDM1 DNA & Klebsiella pneumoniae C099 & 44,859 & LC613145.1 & Thailand \\
\hline pCRE10.4 & Escherichia coli strain CRE10 & 41,191 & CP034403.1 & Thailand \\
\hline plasmid pTR3 & Klebsiella pneumoniae & 41,187 & JQ349086.2 & Singapore \\
\hline pC2974-6-NDM & Klebsiella pneumoniae strain C2974 & 51,995 & CP039800.1 & China \\
\hline pEcloNH77 & Enterobacter cloacae strain NH77 & 41,179 & CP040826.1 & Thailand \\
\hline plasmid pECL189-4 & Enterobacter hormaechei strain 189 & 41,439 & CP047969.1 & China \\
\hline
\end{tabular}

The plasmid pAMA003584_NDM-1 has accession number MZoo4973. Plasmids identified had 91-96\% query coverage and 99.9-100\% identity hits.

ertapenem, but susceptible to meropenem and harboured the blaNDM-1 gene on a $53 \mathrm{~kb}$ Inc $\mathrm{X}_{3}$ plasmid nearly identical (99.7\%) to an IncX3-type blaNDM-1 plasmid from a Raoultella planticola detected in China [13]. During 2019, only a single carbapenemresistant Salmonella Typhimurium var. 0:5-negativecarrying blaOXA-48 was reported in the EU/EEA. In 2018, two isolates of Salmonella Kentucky (OXA-48producing), and single isolates of Salmonella Corvallis (OXA-48-producing), Salmonella Rissen (KPCproducing) and Salmonella Typhimurium (VIM-producing) were identified, whereas no NDMproducing Salmonella were reported [14].

In the case presented here, we identified three bacteria in the same patient, all of which harboured the same carbapenem-resistance gene. Carbapenemase acquisition by an NTS from other Enterobacteriaceae in immunocompromised patients in a healthcare context has been suggested [6]. According to the patient's partner, other guests at the resort also had diarrhoea; we can speculate that this patient may have eaten contaminated food at the resort in Greece, but the exact source of the blaNDM-1 plasmid remains unknown. Furthermore, we can only hypothesise which of the three bacteria first obtained the blaNDM-1 plasmid. Denmark has a low prevalence of carbapenemase-producing bacteria [15] and the fact that $S$. Kottbus is very rare in Denmark also suggests that the three bacteria had 'spent time together' in vivo. During the autumn, the patient was hospitalised and diagnosed with terminal lung cancer. He received different antibiotic regimens so, on the other hand, we cannot rule out an acquisition of the IncN2 plasmid by one of the three bacteria in Denmark.

\section{Conclusions}

We describe the first case of blaNDM-1-positive $S$. Kottbus located on a $43 \mathrm{~kb}$ IncN2-plasmid from an 82 -year-old man with terminal lung cancer detected in
Denmark. The plasmid was also found in E. coli and C. freundii from the same patient, suggesting horizontal gene transfer. The patient had no known travel history outside Europe and could be the first confirmed case of blaNDM-1-positive Salmonella not related to travel outside Europe. Our finding underscores the importance of remaining vigilant for the potential risk of emerging resistance strains. In sum, we find any potential spread of NDM-1-producing NTS worrisome and emphasize the need for antimicrobial resistance surveillance in Europe, especially in countries where NDM-producing Enterobacteriaceae is spreading.

\section{Conflict of interest}

None declared.

\section{Author contributions}

HLN and PKT made the isolation of S. Kottbus and reported the results to the treating physician. AKCS informed the patient and his partner and obtained written informed consent for publication from the latter. MT made the serotyping and SOP, FH and HH made all the WGS-analysis, including identification of the blaNDM-1 plasmid. AKSC and EL made the notification for the Danish health authorities. HLN drafted the manuscript, and all authors revised the manuscript critically and approved the final version.

\section{References}

1. Toboldt A, Tietze E, Helmuth R, Junker E, Fruth A, Malorny B. Molecular epidemiology of Salmonella enterica serovar Kottbus isolated in Germany from humans, food and animals. Vet Microbiol. 2014;170(1-2):97-108. https://doi.org/10.1016/j. vetmic.2014.01.020 PMID: 24559660

2. Centers for Disease Control and Prevention (CDC). Outbreak of Salmonella serotype Kottbus infections associated with eating alfalfa sprouts--Arizona, California, Colorado, and New Mexico, February-April 2001. MMWR Morb Mortal Wkly Rep. 2002;51(1):7-9. PMID: 11831433

3. Enkelmann J, von Laer A, Simon S, Fruth A, Lachmann R, Michaelis K, et al. Disentangling outbreaks using wholegenome sequencing: concurrent multistate outbreaks of Salmonella Kottbus in Germany, 2017. Epidemiol Infect. 
2020;148:e51. https://doi.org/10.1017/So950268820000394 PMID: 32052718

4. Palmera-Suárez R, García P, García A, Barrasa A, Herrera D, Investigation Team. Salmonella Kottbus outbreak in infants in Gran Canaria (Spain), caused by bottled water, AugustNovember 2006. Euro Surveill. 2007;12(7):E070712.2. https:// doi.org/10.2807/esw.12.28.03235-en PMID: 17868561

5. Ryder RW, Crosby-Ritchie A, McDonough B, Hall WJ 3rd. Human milk contaminated with Salmonella kottbus. A cause of nosocomial illness in infants. JAMA. 1977;238(14):1533-4. https://doi.org/10.1001/jama.1977.03280150103039 PMID: 578222

6. Fernández J, Guerra B, Rodicio MR. Resistance to carbapenems in non-typhoidal Salmonella enterica serovars from humans, animals and food. Vet Sci. 2018;5(2):40. https://doi. org/10.3390/vetsci5020040 PMID: 29642473

7. The European Committee on Antimicrobial Susceptibility Testing (EUCAST). Clinical breakpoints and dosing of antibiotics. Växjö: EUCAST. [Accessed: 29 Nov 2020]. Available from: http://www.eucast.org/clinical_breakpoints/

8. Jenkins S, Ledeboer NA, Westblade LF, Burnham CA, Faron ML, Bergman Y, et al. Evaluation of NG-test carba 5 for rapid phenotypic detection and differentiation of five common carbapenemase families: results of a multicenter clinical evaluation. J Clin Microbiol. 2020;58(7):58. https://doi. org/10.1128/JCM.00344-20 PMID: 32376668

9. Wick RR, Judd LM, Gorrie CL, Holt KE. Unicycler: Resolving bacterial genome assemblies from short and long sequencing reads. PLOS Comput Biol. 2017;13(6):e1005595. https://doi. org/10.1371/journal.pcbi.1005595 PMID: 28594827

10. Carattoli A, Zankari E, García-Fernández A, Voldby Larsen M, Lund $\mathrm{O}$, Villa $\mathrm{L}$, et al. In silico detection and typing of plasmids using Plasmidfinder and plasmid multilocus sequence typing. Antimicrob Agents Chemother. 2014;58(7):3895-903. https:// doi.org/10.1128/AAC.02412-14 PMID: 24777092

11. European Food Safety Authority; European Centre for Disease Prevention and Control. The European Union One Health 2019 Zoonoses Report. EFSA J. 2021;19(2):e06406. https://doi. org/10.2903/j.efsa.2021.6406 PMID: 33680134

12. Savard P, Gopinath R, Zhu W, Kitchel B, Rasheed JK, Tekle T, et al. First NDM-positive Salmonella sp. strain identified in the United States. Antimicrob Agents Chemother. 2011;55(12):59578. https://doi.org/10.1128/AAC.05719-11 PMID: 21968356

13. Day MR, Meunier D, Doumith M, de Pinna E, Woodford N, Hopkins KL. Carbapenemase-producing Salmonella enterica isolates in the UK. J Antimicrob Chemother. 2015;70(7):2165-7. https://doi.org/10.1093/jac/dkv075 PMID: 25795771

14. European Food Safety Authority; European Centre for Disease Prevention and Control. The European Union Summary Report on Antimicrobial Resistance in zoonotic and indicator bacteria from humans, animals and food in 2018/2019. EFSA J. 2021;19(4):e06490. https://doi.org/10.2903/j.efsa.2021.6490 PMID: 33868492

15. DANMAP. Use of antimicrobial agents and occurrence of antimicrobial resistance in bacteria from food animals, food and humans in Denmark. ISSN 1600-2032. Copenhagen: Statens Serum Institut, National Food Institute, Technical University of Denmark; 2018. Available from: www.danmap.org

\section{License, supplementary material and copyright}

This is an open-access article distributed under the terms of the Creative Commons Attribution (CC BY 4.0) Licence. You may share and adapt the material, but must give appropriate credit to the source, provide a link to the licence and indicate if changes were made.

Any supplementary material referenced in the article can be found in the online version.

This article is copyright of the authors or their affiliated institutions, 2021. 